\title{
Calculate the score of a social media profile and posts on twitter and other social media sites on the internet
}

\author{
Pooja Chaudhary ${ }^{\text {[0000-0003-4613-8921 ] }}$, Ravin $\operatorname{Kumar}^{\text {[0000-0002-3416-2679] }}$ \\ Department of Computer Science, Meerut Institute of Engineering and Technology, \\ meerut-250005, Uttar Pradesh, INDIA. \\ poojachaudhary831@gmail.com , ravin.kumar.cs.2013@miet.ac.in
}

\begin{abstract}
In the age of social networking, finding the worth of one's social presence and online interactions can have a significant impact on tackling the problem of fake news. We are proposing a scoring mechanism which can also be used to calculate the worth of one's online presence, and also to suggests numerical score to a particular thought posted on the internet.
\end{abstract}

Keywords: twitter, social score, influence score, worth of your post, fake news

\section{Introduction}

Internet has made humans highly interconnected and have fastened the process of sharing ideas, information, and thoughts like never before. This interconnected network can also be used to spread lies [1], spamming [2], sell a perception to the world, and make people believe in a specific type of ideology. In this research paper, we are proposing a method to evaluate the score of any social media interaction including scores for the social account, and posts. These scores can be used in identifying the accounts with the most probability of being spam. Although, these scores can also be found helpful in finding the most important post from the set of reviews posted online, and can also be used to judge the authenticity of the message posted is coming from actual people and not from spam or fake news spreading bots [3].

\section{Proposed Mechanism}

Our proposed mechanism utilizes a self-development algorithm which utilises features such as how many followers does an account has, how many other accounts does it follows, along with other features to calculate a social score. This score suggests the importance or influence ability of that account within its circle.

To better understand our proposed mechanism, let us first look at the description of the variables presented in Table 1. 
Table 1 : Parameters Related Details

\begin{tabular}{|c|c|c|}
\hline S.NO & Parameter & Details \\
\hline 1 & user_id & Unique id for each person's account \\
\hline 2 & FOLLOWERS(id),FRIENDS (id) & Return list of ids of followers and friends respectively. \\
\hline 3 & zeros_list(Z) & Return a list of length Z containing 0 elements. \\
\hline 4 & LENGTH( List ) & Returns number of elements present in a list. \\
\hline
\end{tabular}

Our algorithm does require the degree_of_score to be a non-negative integer. It is used to set how deep one wants to go to calculate the score. Higher the value, more the number of relations (i.e. followers-friend, followers-followers) required to calculate the score for a "user_id".

Function get_zeroscore( user_id )

START

return LENGTH( FOLLOWERS( user_id ) ) / ( LENGTH(FRIENDS( user_id ) ) + 1)

END

Function get_multiscore( list_of_ids, degree_of_score )

START

IF degree_of_score == 1

net_score $=0$

FOR element_id in list_of_ids

net_score $=$ net_score + get_zeroscore $($ element_id $)$

net_score $=$ net_score $/$ LENGTH( list_of_ids )

return net_score

ELSE

degree_of_score $=$ degree_of_score - 1

tmp_list = zeros_list( LENGTH ( list_of_ids ) )

FOR elem_idx in RANGE( 0 , LENGTH ( list_of_ids ) )

new_list_ids $=$ FOLLOWERS $($ list_of_ids [elem_idx] )

tmp_list [elem_idx] = get_multiscore( new_list_ids, degree_of_score )

fin_val $=0$

FOR elem in tmp_list

fin_val $=$ fin_val + elem

fin_val = fin_val / LENGTH( tmp_list)

return fin_val

END 


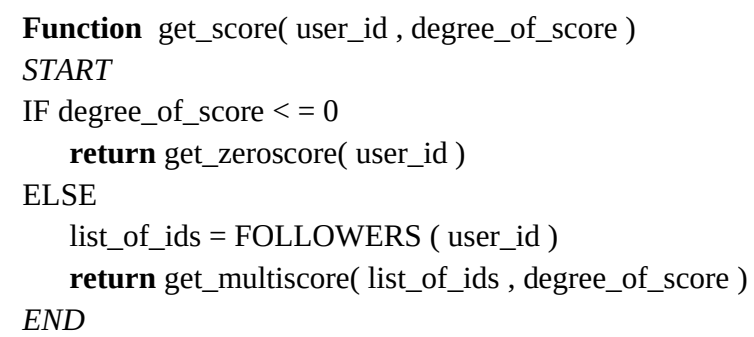

Function Social_Score ( user_id , degree_of_score )

START

delta = get_zeroscore( user_id )

score $=$ get_score $($ user_id, degree_of_score )

return delta * score

END

To calculate the score for a person with id "user_id" at the degree of “degree_of_score” we simply pass these parameters to the function Social_Score.

Score = Social_Score( user_id , degree_of_score )

\section{Working Example}

We have provided open-source code for calculating the social_score [4]. We have also tested it on the twitter platform for the better analysis of our proposed algorithm. Due to the security reasons, we have changed the usernames in the results shown in Table 2.

Higher the calculated score, higher the influence or importance of that user within its group or friend and follower circle. The proposed scoring mechanism can be also used to score a conversation by averaging the score of all the users who participated during the conversation. Similarly, the effect of the agreement or disagreement within the conversation can also be calculated using separately calculating scores for members with agreement and members with disagreement. Later, those two scores can be used to analyze the agreement and disagreement influence in that conversation. 
Table 2 : Experimental Data with degree_of_score = 1

\begin{tabular}{|l|l|l|l|l|}
\hline S.no & User_id & Followers & Friends & Calculated Score \\
\hline 1 & ABC & 9929 & 129 & 60.0663 \\
\hline 2 & DEF & 0 & 1000 & 0 \\
\hline 3 & GHI & 1000 & 0 & 11.83 \\
\hline 4 & JKL & 55 & 21 & 0.96567 \\
\hline 5 & MNO & 2 & 2 & 0.84313 \\
\hline 6 & PQR & 4.2 Million & 2228 & 317 \\
\hline 7 & STU & 111 & 40 & 68.2 \\
\hline 8 & VWX & 100 & 100 & 0.043 \\
\hline 9 & YZA & 52 & 30 & 0.001 \\
\hline 10 & BCD & 16000 & 96 & 185.72 \\
\hline
\end{tabular}

One can also use parallel computing assisted with an effective scheduling mechanism [5] to simultaneously calculate the social score of large number of accounts and then analyze the obtained scores to compare the influence one a member within its friends and follower circle.

\section{Conclusion}

Our proposed scoring mechanism has a wide range of applicability. It can be used to provide the relevance of one's social profile. The scoring mechanism can also be used to find the importance of a post in a conversation, or even the importance of the conversation as a whole. This score can be used to easily eliminate or detect the spamming accounts, and even detect followers increasing bots.

\section{References}

1. Gupta, A., Lamba, H., Kumaraguru, P. and Joshi, A., 2013, May. Faking sandy: characterizing and identifying fake images on twitter during hurricane sandy. In Proceedings of the 22nd international conference on World Wide Web (pp. 729-736). ACM.

2. Thomas, K., Grier, C., Song, D. and Paxson, V., 2011, November. Suspended accounts in retrospect: an analysis of twitter spam. In Proceedings of the 2011 ACM SIGCOMM conference on Internet measurement conference (pp. 243-258). ACM. 
3. Thomas, K., McCoy, D., Grier, C., Kolcz, A. and Paxson, V., 2013. Trafficking Fraudulent Accounts: The Role of the Underground Market in Twitter Spam and Abuse. In Presented as part of the 22nd \{USENIX\} Security Symposium (\{USENIX\} Security 13) (pp. 195-210).

4. Github repository, https://github.com/mr-ravin/social_score , last accessed 2020/8/17.

5. Kumar R. (2019) Cyclic Scheduling Algorithm. In: Shetty N., Patnaik L., Nagaraj H., Hamsavath P., Nalini N. (eds) Emerging Research in Computing, Information, Communication and Applications. Advances in Intelligent Systems and Computing, vol 882. Springer, Singapore. https://doi.org/10.1007/978-981-13-5953-8_38 